\title{
What characterizes the reminiscence bump in autobiographical memory? New answers to an old question
}

\author{
Tabea Wolf ${ }^{1} \cdot$ Daniel Zimprich ${ }^{1}$ \\ Published online: 24 January 2020 \\ (C) The Psychonomic Society, Inc. 2020
}

\begin{abstract}
The reminiscence bump represents one of the most robust findings in autobiographical memory research. As such, it has led to a number of different explanatory accounts that aim to elucidate it. Because most of these accounts have received some empirical support, it has been assumed that they may equally contribute to the explanation of the reminiscence bump phenomenon. In the present study, we used a multilevel multinomial mixed-effects model to examine the predictive power of explanatory variables selected from different accounts simultaneously. Analyses were based on 2,813 autobiographical memories that 97 older adults aged between 60 and 88 years reported in response to 31 cue words. Overall, the predictor variables (i.e., first-time experience, importance and emotional valence) meaningfully distinguished memories from the reminiscence bump from memories from life periods before and after. These effects, however, did not always go into the hypothesized directions. In addition, results of a Commonality Analysis indicated that although the explanatory accounts considered in the present study draw on qualities of autobiographical memories (within-person effects), they might be more useful in explaining why individuals differ in the number of autobiographical memories reported from the reminiscence bump period (between-person effects). Taken together, our findings are in line with a more integrative view on the reminiscence bump that, additionally, emphasizes the individual (e.g., the life-story account).
\end{abstract}

Keywords Reminiscence bump $\cdot$ Autobiographical memory $\cdot$ Multilevel multinomial model $\cdot$ Life-story account

\section{Introduction}

One of the most robust findings in autobiographical memory research is that adults remember a larger number of events experienced during their adolescent and early adult years compared to the life periods before and after. This reminiscence bump has been replicated numerous times (e.g., Alea, Ali, \& Marcano, 2014; Hyland \& Ackerman, 1988; Jansari \& Parkin, 1996; Janssen, Chessa, \& Murre, 2005; Kawasaki, Janssen, \& Inoue, 2011; Rubin \& Schulkind, 1997a, 1997b; Rubin, Wetzler, \& Nebes, 1986; Wolf \& Zimprich, 2016a). In fact, the reminiscence bump has been observed so frequently and under such a broad range of different cueing material that it has been discussed as the defining characteristic of autobiographical memory (Koppel \&

Electronic supplementary material The online version of this article (https://doi.org/10.3758/s13421-019-00994-6) contains supplementary material, which is available to authorized users.

Tabea Wolf

tabea.wolf@uni-ulm.de

1 Developmental Psychology, Institute of Psychology and Education, Ulm University, Albert-Einstein-Allee 47, 89081 Ulm, Germany
Berntsen, 2015). Different accounts have been postulated in order to explain the reminiscence bump phenomenon (e.g., Glück \& Bluck, 2007; Pillemer, 2001; Rubin \& Berntsen, 2003; Rubin, Rahhal, \& Poon, 1998), most of which have received some empirical support. This has led to the assumption that the mechanisms postulated in these accounts may simultaneously contribute to the formation of the reminiscence bump (Demiray, Gülgöz, \& Bluck, 2009; Munawar, Kuhn, \& Haque, 2018). To date, however, only few studies have considered examining different explanatory accounts in the same study (e.g., Demiray et al., 2009; Janssen \& Murre, 2008; Wolf \& Zimprich, 2016a), and it remains an open question to what extent each of these accounts contributes to the understanding of the reminiscence bump phenomenon - once other accounts are also taken into consideration. In the present study, we aimed at closing this gap by testing the predictive power of different explanatory accounts simultaneously.

\section{Theoretical accounts of the reminiscence bump phenomenon}

When autobiographical memories are plotted in terms of the number of memories encoded at different ages across the 
lifespan, typically a specific pattern emerges: Experiences from the recent past (approximately 5 years back in time) are most frequently recalled (recency effect), and the number of autobiographical memories then declines from the present back to the period of young adulthood. Up to that point, the recall of autobiographical memories resembles typical forgetting curves found in research on episodic memory, where recall performance decreases non-linearly across retention time (e.g., Zimprich \& Kurtz, 2013). Unlike episodic memory forgetting curves, the number of autobiographical memories increases again once the period of one's younger adulthood is reached and peaks around a person's adolescent years (reminiscence bump). Rubin et al. (1986) were among the first to systematically analyze this lifespan retrieval curve of autobiographical memories. They found an increase in autobiographical memories from the time when participants were aged between 10 and 30 years. Since then, the reminiscence bump has been replicated numerous times for autobiographical memories elicited with the cue-word technique (e.g., Alea et al., 2014; Hyland \& Ackerman, 1988; Jansari \& Parkin, 1996; Janssen et al., 2005; Kawasaki et al., 2011; Rubin \& Schulkind, 1997a, 1997b; Wolf \& Zimprich, 2016a). Reminiscence bumps were also found for autobiographical memories elicited under more or less restrictive recall instructions, for example, for vivid or important autobiographical memories (e.g., Ece \& Gülgöz, 2014; Fitzgerald, 1988; Glück \& Bluck, 2007; Rubin \& Schulkind, 1997a; Webster $\&$ Gould, 2007), or free (uncued) recall from different periods of participants' lives (e.g., Conway, Wang, Hanyu, \& Haque, 2005; Demiray et al., 2009). Moreover, reminiscence bumps have been found for preferences in cultural life such as literature, film, or music (Holbrook \& Schindler, 1989; Janssen et al., 2007; Larsen, 1996; Rathbone, O'Connor, \& Moulin, 2017; Zimprich, 2018; Zimprich \& Wolf, 2016b).

Different theoretical accounts have been postulated to explain the reminiscence bump phenomenon. These accounts share the assumption that there is something special with regard to autobiographical memories stemming from adolescence and young adulthood. Most of them focus on characteristics of the events experienced during one's youth. For instance, the cognitive explanatory account (Rubin et al., 1998) argues in favor of a preferential encoding of novel events. Compared to familiar experiences, novel events may entail greater cognitive efforts in order to understand and integrate them. Thus, novel events may benefit from a deeper processing, which entails a better encoding and retrieval (Craik \& Lockhart, 1972). In addition, first-time experiences are more distinct than repeated events and thus may lead to more distinctive memory traces (Robinson, 1992), which, again, increases their recallability (e.g., Eysenck \& Eysenck, 1980). The first experience of an event is also more likely to function as an exemplar for similar experiences encountered later in life (Janssen, Rubin, \& St. Jacques, 2011; Pillemer, 2001), which also enhances their recallability (Bjork, 1975). Because adolescence and young adulthood are associated with a greater number of first-time experiences (e.g., the first kiss, the first relationship, or the first job), this could explain the increased recall of memories from these life periods. Jansari and Parkin (1996; Experiment 2), for example, examined whether memories from different life periods differed regarding the number of firsttime experiences. They found that memories from an early life period (from the age of 0 to 20 years) included a higher proportion of first-time experiences than memories from the recent past (10-20 years ago). Moreover, the early first-time experiences were more easily retrievable (i.e., had shorter retrieval times) compared to first-time experiences from the more recent past or non-first-time experiences. Taken together, these findings provide support for the assumption that the reminiscence bump may be due to a preferential encoding of first-time experiences (see also Demiray et al., 2009).

The identity-formation or narrative account (Conway \& Haque, 1999; Fitzgerald, 1988) draws on the role of autobiographical memory in forming and maintaining a sense of selfidentity. According to theories on human development, adolescence and early adulthood are associated with the development of a personal identity (e.g., Erikson, 1950). Such a sense of identity relies on integrating different personal experiences into a coherent self-narrative (Fitzgerald, 1988, 1996; Prebble, Addis \& Tippett, 2013). Experiences from the life period in which a person's identity was developed may be recalled frequently and remain highly accessible due to their ongoing relation to the self (Conway, 2005; Rathbone et al., 2008). Support for this assumption was found in a study conducted by Demiray et al. (2009), who asked participants to rate the importance of autobiographical memories for the development of their identity. Comparing the average ratings of memories falling between the ages of 10 and 30 years (i.e., the reminiscence bump period), they found these memories to be significantly more important for identity development than memories from outside the reminiscence bump period. Additional support comes from studies that found a reminiscence bump in the distribution of participants' most important autobiographical memories (e.g., Berntsen \& Rubin, 2002; Glück \& Bluck, 2007)

Another account of the reminiscence bump phenomenon is the so-called cultural life-script account (Berntsen \& Rubin, 2002). In contrast to previous accounts that mainly focus on differential encoding and retention of memories experiences during adolescence and early adulthood, the life script account puts emphasis on the retrieval of autobiographical memories. Berntsen and Rubin (2002) argued that individuals have an internalized representation of what persons typically experience throughout their lives, as well as the time at which these events are most likely to occur. When asked to recall autobiographical memories, these culturally shared life scripts may structure the retrieval processes. Because the majority of life-scripted events are located in young adulthood (e.g., graduation, marriage, and childbirth), events from this life period 
are most frequently recalled. Additionally, the life-script account predicts an association between the emotional quality of events and the reminiscence bump: Although cultural life scripts can, of course, include negative events, they have been found to contain higher proportions of positive life events and may thus favor the retrieval of emotionally positive autobiographical memories (e.g., Berntsen \& Rubin, 2004; Ece \& Gülgöz, 2017; Zaragoza Scherman, 2013). For instance, Berntsen and Rubin (2002) reported a reminiscence bump in the distribution of participants' happiest as well as for their most important memories, whereas the distributions of participants' saddest and most traumatic memories showed a monotonically decreasing retention function (from the present back) aggregated across persons. With respect to the most positive and the most stressful or traumatic memories, these findings were replicated in participants from Mexico, China, Greenland, and Denmark (Zaragoza Scherman, Salgado, Shao, \& Berntsen, 2015). Thus, although the life-script account focuses on retrieval processes, it also allows for predictions regarding the characteristics of the memories recalled (i.e., an emotionally positive valence).

The explanatory accounts of the reminiscence bump mentioned are not mutually exclusive (e.g., Rubin et al., 1998); they each focus on different qualities of autobiographical memories such as the novelty of events (i.e., first-time experiences), their importance for one's life and identity, and whether they refer to positive life-scripted events. These different qualities were integrated in the more recently proposed life-story account (Glück \& Bluck, 2007), which takes a lifespan developmental perspective on the reminiscence bump phenomenon. Classical lifespan developmental theories posit that people in different life phases have to cope with different developmental tasks (e.g., Erikson, 1950; Havighurst, 1948). As outlined above, adolescence and the early adult years are associated with the development of a person's identity. In addition, young adults start to take control over their lives by making decisions about their professional and personal future. Many of the events related to these decisions are part of culturally shared life-scripts (e.g., starting a new job, getting married, etc.). By marking the beginning of a new period in a person's life, these decisions are also likely to entail a number of first-time experiences. Finally, these decisions do not only concern a person's current life situation but may actually affect his or her entire adult life (Arnett, 2000; Ebner, Freund, \& Baltes, 2006). Due to their ongoing influence on development, they remain highly accessible throughout the lifespan as part of a person's individual life story. Taken together, the lifestory account combines previous accounts by hypothesizing that memories from an individual's youth are "currently viewed as more novel and more important for how one's identity has developed, and (due to lifespan developmental influences) are likely to be more distinct and to include developmental transitions than memories from other life phases"
(Demiray et al., 2009, p. 722). Support for this more integrative view on the reminiscence bump raises from the aforementioned study by Demiray et al. (2009). The authors compared autobiographical memories from the reminiscence bump period (between the ages of 10 and 30 years) with memories from outside the bump regarding novelty, importance for identity development, and transition. Taken together, their findings supported the more integrative view of the life-story account by showing that memories from the reminiscence bump period include more first-time experiences and transitional events than memories from outside the bump. In addition, autobiographical memories from the reminiscence bump period were, on average, perceived as more important for identity development (see also Glück \& Bluck, 2007).

\section{Testing explanatory accounts of the reminiscence bump simultaneously}

Different theoretical accounts that aim to explain the reminiscence bump phenomenon have received some empirical support (see Munawar et al., 2018, for a detailed review). Although it is widely understood that these accounts are not mutually exclusive (e.g., Ece \& Gülgöz, 2017; Koppel \& Berntsen, 2015; Rubin et al., 1998), only a few studies have considered examining different explanatory accounts in the same study one by one (e.g., Demiray et al., 2009; Janssen \& Murre, 2008). What is still missing in the literature, however, is a simultaneous test of different explanatory accounts. Such a simultaneous test is inherently multivariate. To see this, one has to keep in mind that a single autobiographical memory shows qualities that fit into more than one explanatory account. For example, an autobiographical memory might describe a first-time experience, might have positive emotional valence, and might be considered important. In other words, every autobiographical memory a participant reports can be described with respect to several "explanatory" variables simultaneously. This necessarily implies that explanatory or predictor variables are correlated. For this reason, examining several variables one by one overestimates the explanatory power of an individual variable - the amount of overestimation will be larger the stronger an explanatory variable is correlated with other explanatory variables.

One way to disentangle the effects of correlated predictor variables on an outcome variable is commonality analysis (e.g., Nimon \& Reio, 2011; Ray-Mukherjee et al., 2014), which we will use in the present article (see below). Briefly, a commonality analysis decomposes the total effects of several predictor variables examined simultaneously into its unique and common effects. Unique effects indicate how much variance in the outcome variable is uniquely accounted for by an individual predictor variable. By contrast, common effects indicate how much variance in the outcome variable is accounted for by predictor variables jointly. Transferred to 
the goal of the present article, that is, testing different explanatory accounts of the reminiscence bump simultaneously, a commonality analysis offers important insights: (1) The unique contribution would indicate the "net" effect of an explanatory variable, that is, the effect that only this explanatory account explains in the reminiscence bump after controlling for other explanatory accounts. (2) The common contribution would indicate how much the different explanatory variables overlap empirically in the sense that they jointly account for the reminiscence bump. Both are important in evaluating explanatory accounts of the reminiscence bump.

These analyses can be based on (at least) two different levels. In studies that examine the reminiscence bump phenomenon, participants are typically instructed to recall a given number of autobiographical memories. If participants provide more than one autobiographical memory, the resulting data have a hierarchical, clustered, or multilevel structure in the sense that autobiographical memories (Level 1) are nested within participants (Level 2). Because explanatory accounts of the reminiscence bump mainly focus on qualities of autobiographical memories (e.g., first-time experiences, importance and emotional valence), they naturally lead to predictions on Level 1, which refers to characteristics of the memories. These accounts may in addition lead to predictions on Level 2, which refers to characteristics of the participants. To give an example, based on the cognitive explanatory account, one would expect that autobiographical memories describing first-time experiences have a higher likelihood of falling into the reminiscence bump period than into life periods before and after. Because autobiographical memories are nested within participants, Level 1 represents the within-person level and captures differences between autobiographical memories. An analysis on Level 1 could then, for example, lead to the finding that, in accordance with the cognitive explanatory account, those autobiographical memories describing first-time experiences are more likely to stem from the reminiscence bump period of the participants' lives.

Level 2 represents the between-person level because it captures differences between participants. What distinguishes variables located on Level 2 from variables located on Level 1 is that the Level 2 variables are constant across Level 1 units (autobiographical memories) or, equivalently, constant within persons. Although most explanatory accounts focus on characteristics of autobiographical memories (Level 1), they also lead to predictions on Level 2. To see this, new individual differences variables can be formed by averaging Level 1 variables within a Level 2 unit to result in so-called "context means" (Bryk \& Raudenbush, 1992) - a common practice in multilevel analyses. Thus, for example, one could calculate the proportion of autobiographical memories describing a first-time experience a participant reported (by dividing the number of autobiographical memories describing first-time experiences a participant reported by the total number of autobiographical memories that participant reported). This proportion of first-time experiences then represents a Level 2 variable, i.e., it is constant within a participant. In further pursuing the cognitive explanatory account of the reminiscence bump, for example, one would expect that those participants showing a higher proportion of first-time experiences, report more autobiographical memories from the reminiscence bump period than participants with a lower proportion of first-time experiences memories. Thus, a commonality analysis might provide new insights on both levels of analysis.

\section{The present study}

The overarching goal of the present study was to examine the predictive power of different explanatory accounts of the reminiscence bump simultaneously and on two levels of analysis. In a first step, we tested three specific predictions derived from different theoretical accounts of the reminiscence bump phenomenon. Specifically, based on the cognitive explanatory account, we expected that an autobiographical memory that describes a firsttime experience is more likely to stem from the reminiscence bump period than from the life periods before or after (Level 1 prediction). At the same time, we expected that participants who show a greater proportion of autobiographical memories describing first-time experiences, report more autobiographical memories from the reminiscence bump (Level 2 prediction). Based on the identity-formation account, we expected that the more an autobiographical memory is considered as important for an individual's life, the greater is the likelihood that this memory would fall into the reminiscence bump period (Level 1 prediction). In addition, we expected that the reminiscence bump becomes more pronounced for persons who perceive their memories, on average, as more important (Level 2 prediction). The life-script account and the life-story account additionally predict an association between the reminiscence bump and emotionally positive memories. Based on these accounts, we expected that the more an autobiographical memory is perceived as positive, the greater is the likelihood that this memory would fall into the reminiscence bump period (Level 1 prediction). Regarding Level 2, we expected that individuals who, on average, report emotionally more positive autobiographical memories recall (relatively) more memories from the reminiscence bump. In contrast to previous studies (e.g., Demiray et al., 2009; Janssen \& Murre, 2008), we examined the effect of each predictor variable while controlling for possible effects of the other.

In a second step, we evaluated the predictive power of these explanatory variables using commonality analysis. Apart from disentangling the unique contribution of each explanatory variable (after controlling for other explanatory variables), we aimed at examining the extent to which different explanatory variables overlap empirically. Although there is a general agreement in that different explanatory accounts are not mutually exclusive - or may even contribute simultaneously to the reminiscence bump 
phenomenon (e.g., Demiray et al., 2009; Ece \& Gülgöz, 2017; Koppel \& Berntsen, 2015; Rubin et al., 1998), this assumption has not been tested adequately yet, that is, based on a multivariate analytical approach (and on two different levels of analysis). Thus, the present result can provide new insights on the frequently asked question of what characterizes the reminiscence bump in autobiographical memory.

\section{Methods}

\section{Participants}

The sample comprised a total of 97 older adults from Germany (48 women and 49 men). Participants were aged between 60 and 88 years $\left(M_{\text {age }}=68.18\right.$ years, $\left.S D=6.11\right)$. The sample had 17 participants (18\%) reporting to have graduated from university and 11 participants (11\%) graduated from high school. Note that in Germany, a high school degree involves approximately 13 years of education.

On a scale ranging from 1 (excellent) to 5 (poor), subjective health was judged as 2.33 , on average $(S D=1.01)$. Forty-five participants (46\%) reported suffering from a chronic condition. The chronic condition most often mentioned was hypertension (27 out of 45 participants with a chronic condition). Importantly, no participant reported suffering from a neurological disease.

\section{Procedure}

Older adults were interviewed individually. After giving their informed consent, participants provided demographic (e.g., age, gender, and education) and health information. Subsequently, participants were presented with a cue word printed on a small card (31 cue words in total), and were asked to report an autobiographical memory that came to their mind. The order of cue words was random. Participants were instructed to report events that were at least ten years old to avoid the recency effect often found in autobiographical memory. Our decision to exclude the recency effect was based on the rationale that more recent memories may be qualitatively different from long-term AMs because their distribution closely resembles a forgetting function (where accessibility decreases with the passage of time). Moreover, it remains an open question whether recent memories ever (and if so, which of them) turn into long-term AMs that can be accessed many years later.

After participants had shortly described the memory, they were asked whether it described an event or experience that had happened for the first time. Subsequently, participants were required to judge the emotional valence of the reported event on a scale ranging from 1 (very negative) to 7 (very positive). Next, the subjective importance of an event for the participant's life was rated on a 5-point Likert-type scale ranging from 1 (not at all important) to 5 (very important). Afterwards, the next cue word was presented. After all events had been described, the interviewer went through the recalled events again and asked the participants for their age at the time of the event. All cue words were selected from the Berlin Affective Word List (BAWL-R; Võ et al., 2009) with the constraint that words were high in imageability and emotionally neutral.

\section{Statistical modeling}

We used a multilevel multinomial mixed-effects model as described by Zimprich and Wolf (2018). A multinomial regression allows for modelling the probability of the (unordered) categories of a (dependent) variable. Transferred to the goals of our study, we can examine whether explanatory variables change the probability of memories falling into the life period before the reminiscence bump - which one might refer to as the category of Pre-Bump memories - and whether explanatory variables change the probability of memories falling into the life period after the reminiscence bump - which one might refer to as the category of Post-Bump memories. Because probabilities must add up to one, the probability of a memory falling into the reminiscence bump - which one might refer to as the category of Bump memories - is given as one minus the Pre-Bump and Post-Bump probabilities. The multinomial regression model can then be thought of as simultaneously estimating logistic regression models for the comparisons between (1) the Pre-Bump versus Bump category and between (2) the Bump and Post-Bump category of autobiographical memories.

Because the autobiographical memory data in our study exhibit a two-level structure (Level 1: autobiographical memories, Level 2: individuals), we extended the multinomial regression model by random intercept effects to take the hierarchical nature of the data into account (see Hedeker, 2003; Hedeker \& Gibbons, 2006). A detailed description of this procedure is given in Zimprich and Wolf (2018). SAS PROC NLMIXED syntax for the multinomial mixed-effects model is available from the authors upon request.

In a first step, we created three categories of autobiographical memories based on the age at which the event or experience described in the autobiographical memory took place. To foreshadow results, based on Fig. 1 we defined the reminiscence bump in the present study to lie between the age boundaries of 11 and 25 years. Consequently, the following age boundaries were used to define the three categories of Pre-Bump (category A), Bump (B), and Post-Bump (C) memories:

Category A (Pre-Bump): 0 years $<$ Age at $A M \leq 10$ years, Category B (Bump): 11 years $\leq$ Age at $\mathrm{AM} \leq 25$ years, Category C (Post-Bump):26 years $\leq$ Age at AM, 


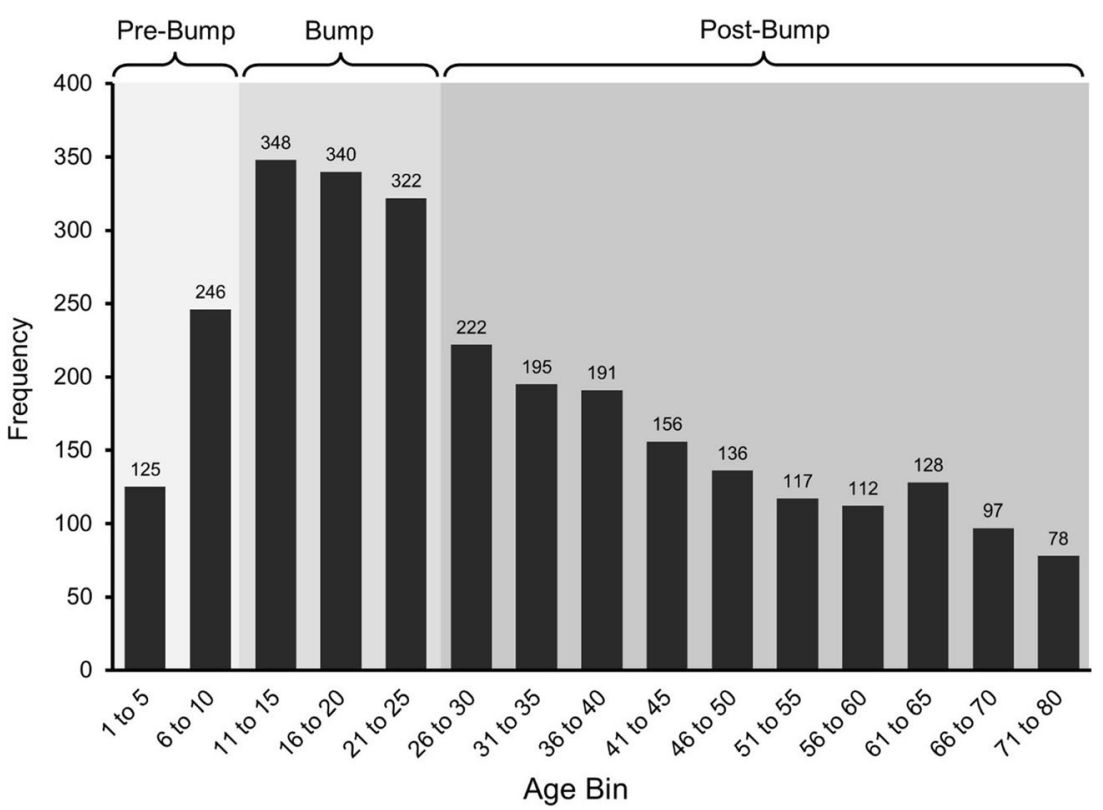

Fig. 1 Distribution of autobiographical memories aggregated into 5-year age bins. The shaded areas represent pre-bump memories $(1 \leq$ age $\leq 10)$, bump memories $(11 \leq$ age $\leq 25)$, and post-bump memories $(26 \leq$ age $)$, respectively

where AM denotes autobiographical memory. ${ }^{1}$

Predictor variables were derived from different explanatory accounts of the reminiscence bump phenomenon. To examine the cognitive account, we used the question of whether an autobiographical memory described a first-time experience. The identity-formation account was tested based on the importance of an event for a person's life. The emotional valence of an event was included to additionally examine the life-script account and the life-story account. Each predictor entered the model on two levels, both as a within-person and a between-person variable. For instance, first-time experiences entered the model on Level 1 to indicate whether the individual autobiographical memory described a first-time experience and on Level 2 as the proportion of autobiographical memories describing a first-time experience. Similarly, importance on Level 1 reflects the importance of an individual autobiographical memory for an individual's life, whereas on Level 2 it reflects the average importance of all autobiographical memories a participant had reported. Likewise, emotional valence on Level 1 reflects the emotional valence of an individual autobiographical memory, whereas on Level 2 it reflects the average emotional valence of the autobiographical

\footnotetext{
${ }^{1}$ Two reviewers wondered how our results would look like if the bump category were defined with age boundaries different from the [11, 25] interval we chose based on the actual autobiographical memories distribution (see Fig. 1). While one reviewer suggested an $[11,30]$ interval for the bump, the other suggested $[9,23]$ as the most probable bump interval. We reran the analyses based on these different bump intervals. The general pattern of results (i.e., statistically significant versus non-significant predictors; see Table 2) was the same with the different bump intervals. Moreover, estimates were numerically rather close (apart from the intercepts).
}

memories a participant generated. Level 1 variables were centered within person (group-mean centered), whereas Level 2 variables were centered across persons (grand-mean centered) - with the result that the within-person and the between-person parts of a predictor variable are independent or uncorrelated. More specifically, if $X_{i j}$ denotes the predictor variable (e.g., emotional valence) measured for autobiographical memory $j$ in participant $i$, withinperson centering was done according to

$X_{i j}^{c}=\left(X_{i j}-\overline{X_{i}}\right)$

where $X_{i j}^{c}$ denotes the variable centered within person $i$ and $\overline{X_{i}}$ denotes the $i$ th participant's mean of the predictor variables across measurements.

One consequence of this type of centering of Level 1 variables is that they (can) only account for Level 1 variance in the outcome variable, whereas Level 2 variables (where the centering is only done for ease of interpretation) only account for Level 2 variance in the outcome variable. Apart from the predictor variables mentioned, we included sex and age as demographic variables to be controlled for. Age was entered grand-mean centered and sex was entered dummy-coded with 0 (male) and 1 (female).

Data were analyzed using SAS NLMIXED (SAS Institute Inc., 2014) using Gauss-Hermite quadrature with 15 quadrature points. As criteria for model fit, the Akaike Information Criterion (AIC; Akaike, 1974) and the Bayesian Information Criterion (BIC; Schwarz, 1978) were used. Both are based on minus two times the log-likelihood of the data given the model in question plus a penalty factor for introducing additional parameters, thus rewarding parsimony - with the AIC penalizing the number of 
parameters less strongly than the BIC. A thorough discussion of the properties of both the AIC and the BIC can be found in Vrieze (2012). In order to decompose the variance accounted for in the outcome variables Pre-Bump (vs. Bump) and PostBump (vs. Bump) into portions attributable uniquely to firsttime experiences, emotional valence, and importance, a commonality analysis was conducted (Nimon \& Reio, 2011; RayMukherjee et al., 2014).

\section{Results}

\section{Descriptive statistics}

Figure 1 shows the distribution of autobiographical memories generated by the 97 participants in the present study, aggregated into 5-year age bins and across participants. Due to missing values (some participants were unable to recall an autobiographical memory to some cue words), there were not 97 (sample size) $\times 31$ (number of cue words) $=3,007$ autobiographical memories in total, but 2,813 autobiographical memories (ranging from 24 to 30 per participant). Based on Fig. 1, we defined the reminiscence bump to fall between the age boundaries of 11 and 25 years, because the corresponding three age bins showed the largest frequencies.

Descriptive statistics are depicted in Table 1. Of the 2,813 autobiographical memories, $35.9 \%$ fell into the Bump category, while $13.2 \%$ and $50.9 \%$ fell into the Pre-Bump and PostBump categories, respectively. Around half of all autobiographical memories were categorized as describing first-time experiences $(48.9 \%)$. The Pre-Bump category included the highest proportion of first-time experiences $(60.7 \%)$, whereas $49.8 \%$ of the Bump and $45.2 \%$ of the post-Bump memories described first-time experiences. The emotional valence of autobiographical memories was 4.2 , on average. Bump memories were rated as most positive, followed by post-Bump and Pre-Bump memories. The importance of memories was 3.07, on average. The average ratings of importance increased from the Pre-Bump over the Bump into the post-Bump category.

On Level 2, results were similar. The mean proportion of first-time experiences decreased from the Pre-Bump over the Bump into the post-Bump category, whereas the mean importance of autobiographical memories showed the reversed pattern, that is, an increase from the Pre-Bump over the Bump into the post-Bump category. With respect to the average emotional valence, Bump memories were the most positive ones followed by Post-Bump memories.

\section{Multilevel multinomial regression models}

In a first model (Model 0), only fixed intercepts were estimated, that is, neither explanatory variables nor random effects were included. Table 2 shows the parameter estimates for the comparison of Pre-Bump (A) with Bump (B) and Post-Bump (C) with Bump (B). The statistically significant intercept estimate for the PreBump category was $\beta_{0 \mathrm{~A}}=-1.002$. For an interpretation of this estimate in terms of probability, two different, but related calculations are helpful. First, if the estimate is transformed back to the probability scale - with the use of the Post-Bump intercept estimate of $\beta_{0 \mathrm{C}}=0.349-$ one gets

Table 1 Descriptive Statistics of Autobiographical Memories (Level 1 and Level 2)

\begin{tabular}{|c|c|c|c|c|c|c|c|c|c|}
\hline \multirow[b]{2}{*}{ Category } & & \multirow[b]{2}{*}{ Total } & \multicolumn{4}{|c|}{ Within Persons (Level 1) } & \multicolumn{3}{|c|}{ Between Persons (Level 2) } \\
\hline & & & $\begin{array}{l}\text { First- } \\
\text { time }\end{array}$ & & Emotional valence & Importance & $\begin{array}{l}\text { First- } \\
\text { time }\end{array}$ & Emotional Valence & Importance \\
\hline \multirow[t]{4}{*}{ Pre-Bump (A) } & $\mathrm{N}$ & 371 & 225 & Mean & 3.98 & 2.78 & 0.52 & 4.17 & 3.01 \\
\hline & $\%$ & 13.2 & 60.7 & $S D$ & 2.01 & 1.07 & 0.10 & 0.70 & 0.19 \\
\hline & & & & Min & 1 & 1 & 0.27 & 3.13 & 2.60 \\
\hline & & & & $\operatorname{Max}$ & 7 & 5 & 0.73 & 4.88 & 3.47 \\
\hline \multirow[t]{4}{*}{ Bump (B) } & $\mathrm{N}$ & 1010 & 503 & Mean & 4.40 & 2.98 & 0.50 & 4.21 & 3.05 \\
\hline & $\%$ & 35.9 & 49.8 & $S D$ & 1.98 & 1.01 & 0.10 & 0.61 & 0.17 \\
\hline & & & & Min & 1 & 1 & 0.29 & 3.55 & 2.71 \\
\hline & & & & $\operatorname{Max}$ & 7 & 5 & 0.81 & 4.92 & 3.65 \\
\hline \multirow[t]{4}{*}{ Post-Bump (C) } & $N$ & 1432 & 647 & Mean & 4.12 & 3.20 & 0.47 & 4.20 & 3.09 \\
\hline & $\%$ & 50.9 & 45.2 & $S D$ & 1.83 & 1.06 & 0.09 & 0.53 & 0.17 \\
\hline & & & & Min & 1 & 1 & 0.12 & 3.11 & 2.51 \\
\hline & & & & Max & 7 & 5 & 0.69 & 4.68 & 3.82 \\
\hline \multirow[t]{2}{*}{ Total } & $N$ & 2813 & 1375 & Mean & 4.20 & 3.07 & 0.49 & 4.20 & 3.06 \\
\hline & $\%$ & 100 & 48.9 & $S D$ & 1.91 & 1.06 & 0.10 & 0.59 & 0.18 \\
\hline
\end{tabular}


Table 2 Models and Parameter Estimates

\begin{tabular}{|c|c|c|c|c|c|c|c|c|}
\hline \multirow[t]{2}{*}{ Model } & \multicolumn{2}{|l|}{0} & \multicolumn{2}{|l|}{1} & \multicolumn{2}{|l|}{2} & \multicolumn{2}{|l|}{3} \\
\hline & Pre-Bump & Post-Bump & Pre-Bump & Post-Bump & Pre-Bump & Post-Bump & Pre-Bump & Post-Bump \\
\hline \multicolumn{9}{|l|}{ Fixed Effects } \\
\hline Intercept & $-1.002 *$ & $0.349^{*}$ & $-1.332 *$ & $0.298 *$ & $-1.226^{*}$ & 0.067 & $-1.309 *$ & 0.067 \\
\hline b: Age & & & & & -0.229 & 0.006 & -0.230 & 0.005 \\
\hline b: $\operatorname{Sex}(0=$ male, $1=$ female $)$ & & & & & $0.431 *$ & -0.229 & $0.441 *$ & -0.216 \\
\hline w: First Time & & & & & & & $0.398 *$ & $-0.172 *$ \\
\hline b: First Time & & & & & & & $2.824 *$ & $-3.504 *$ \\
\hline w: Emotional Valence & & & & & & & $-0.106^{*}$ & $-0.085^{*}$ \\
\hline b: Emotional Valence & & & & & & & 0.006 & -0.145 \\
\hline w: Importance & & & & & & & $-0.186^{*}$ & $0.208 *$ \\
\hline b: Importance & & & & & & & $-1.329 *$ & $1.624 *$ \\
\hline \multicolumn{9}{|l|}{ Random Effects } \\
\hline Intercept Variance & & & $0.583 *$ & $0.772 *$ & $0.605 *$ & $0.748 *$ & $0.514 *$ & $0.593 *$ \\
\hline Intercept Correlation & & & $-0.439 *$ & & $-0.451 *$ & & $-0.377 *$ & \\
\hline \multicolumn{9}{|l|}{ Model Fit } \\
\hline$-2 \mathrm{LL}$ & 5505.9 & & 5121.6 & & 5083.5 & & 4903.4 & \\
\hline$\Delta-2 \mathrm{LL}$ & & & 0384.3 & & 0038.1 & & 0180.1 & \\
\hline AIC & 5509.9 & & 5131.6 & & 5101.5 & & 4945.5 & \\
\hline BIC & 5521.8 & & 5144.5 & & 5124.7 & & 4999.6 & \\
\hline
\end{tabular}

* $p<$.05. Note: "w:" denotes a Level 1 (or within-person) effect, "b:" denotes a Level 2 (or between-person) effect; AIC = Akaike Information Criterion (smaller is better); BIC = Bayesian Information Criterion (smaller is better)

$$
\begin{aligned}
p_{i j \mathrm{~A}} & =\frac{\exp \left(\beta_{0 \mathrm{~A}}\right)}{1+\exp \left(\beta_{0 \mathrm{~A}}\right)+\exp \left(\beta_{0 \mathrm{C}}\right)} \\
& =\frac{\exp (-1.002)}{1+\exp (-1.002)+\exp (0.349)}=0.132
\end{aligned}
$$

implying that $13.2 \%$ of the autobiographical memories are predicted to fall into the Pre-Bump category. For the PostBump intercept estimate, an analogous calculation leads to $p_{i j \mathrm{C}}=0.509$. Therefore, the probability of an autobiographical memory falling into the Bump is $p_{i j \mathrm{~B}}=1-0.132-0.509=$ 0.359 . Because no predictor variables were included in the model, these probabilities exactly equal the observed relative frequencies given in Table 1.

As an alternative, an odds ratio (OR) can be calculated according to

$O R_{i j \mathrm{~A}}=\exp \left(\beta_{0 \mathrm{~A}}\right)=\exp (-1.002)=0.367$

implying that the odds of an autobiographical memory falling into the Pre-Bump category are 0.367 times the odds of an autobiographical memory falling into the Bump category. For the PostBump intercept estimate, an according calculation leads to $\mathrm{OR}_{i j \mathrm{C}}=$ 1.41 , showing that the odds for an autobiographical memory to fall into the Post-Bump category are 1.41 times the odds of the Bump category.
In the next model (Model 1), random effects for the intercepts were added to take into account the hierarchical structure of the data, which led to a considerable increase in model fit (compared to Model 0). As Table 2 shows, random-effects variances were statistically different from zero, implying that participants differed reliably in their proportion of Pre-Bump and Post-Bump autobiographical memories. The intraclass correlation was 0.15 for the Pre-Bump regression, implying that $15 \%$ of the variance in the comparison between the Pre-Bump and Bump categories of autobiographical memories were on Level 2 and, thus, reflected between-person differences. Regarding the comparison between the Post-Bump and the Bump categories, 19\% of the variance reflected between-person differences. ${ }^{2}$ The random effects showed

\footnotetext{
${ }^{2}$ One may wonder why after including random effects in Model 1 the intercept estimates also changed in comparison with Model 0. In generalized linear mixed models, one distinguishes between marginal and conditional models. In contrast to normally distributed data, for non-Gaussian data the observed (or marginal) distribution of the outcome variable and the distribution of the outcome variable conditional on the random effects are not the same (cf. Stroup, 2013). The larger the random variance is, the larger this discrepancy becomes. Thus, in Model 0 the fixed effects estimates represent marginal effects, while in Model 1 fixed effects estimates represent effects conditional on the random effects. A more extensive discussion of marginal versus conditional models can be found in Diggle, Heagerty, Liang, and Zeger (2002), for example. Hedeker, Du Toit, Demirtas, and Gibbons (2018), among others, describe an approach of how marginal probability estimates can be obtained based on parameter estimates from a conditional model.
} 
a negative correlation $(-.44)$, indicating that participants who reported (relatively) more memories from the Pre-Bump category tended to report (relatively) fewer memories from the Post-Bump category (and vice versa).

In Model 2, age and sex were entered as Level 2 demographic variables to be (statistically) controlled for, which, again, led to an increase in model fit (compared to Model 1). As Table 2 shows, age did not have a statistically significant effect. Sex did not affect the Post-Bump category significantly but had a statistically significant effect on the Pre-Bump category in the sense that women reported more autobiographical memories from their childhood years (age 1 to than men. In terms of an odds ratio, one has $\mathrm{OR}=\exp \left(\beta_{2 \mathrm{~A}} \times \operatorname{sex}\right)=\exp (0.431)=1.54$, implying that the odds of a woman (of average age) reporting an autobiographical memory from the Pre-Bump category are about 1.5 times the odds of a man (of average age).

In the final model (Model 3), the Level 1 and Level 2 effects of first-time experiences, importance and emotional valence were included. Compared to Model 2, model fit increased substantially (see Table 2). Regarding the Pre-Bump with Bump comparison, the statistically significant effect of first-time experiences was 0.398 , implying that autobiographical memories describing firsttime experiences are more likely in the Pre-Bump than in the Bump category (OR: 1.49). Emotional valence and importance showed a statistically significant negative effect, implying that emotionally more positive, respectively more important memories were less likely to fall into the Pre-Bump category compared to the Bump category. The ORs of these effects were 0.90 for emotional valence and 0.83 for importance. This implies that (because both are continuous variables) with every unit that the emotional valence (or importance) of an autobiographical memory increases, the odds for this autobiographical memory to fall into the PreBump category as compared to the Bump category decrease by the factor 0.90 (or 0.83). At the between-person level, the effect of first-time experiences and importance became statistically significant. Participants who recalled more first-time experiences recalled significantly more autobiographical memories from the Pre-Bump category compared to the Bump category (OR: 16.84). ${ }^{3}$ In contrast, participants who reported more important memories recalled significantly less memories from the PreBump compared to the Bump category (OR: 0.26).

\footnotetext{
${ }^{3}$ One may wonder why this odds ratio is so large. The reason is the underlying scaling of the variable "proportion of first-time experiences" ("b: First Time" in Table 2), which, as a proportion, has a theoretical range from 0 (a person who reported no first-time experiences at all) to 1 (a person who only reported first-time experiences). Because the regression parameter estimate predicts the change in the outcome variable if the predictor variable is increased by 1 unit, the odds ratio of 16.84 describes the difference in the odds for a person with no and a person with only first-time experiences - these extreme proportions do not occur in the data. One can, however, easily transform the estimate. If, for example, one prefers the effect of a 0.01 increase in the proportion of first-time experiences, one divides the parameter estimate (see Table 2 ) by 100 , that is, $2.824 / 100=0.02824$, and then calculates the odds ratio (OR: 1.028).
}

Turning to the Post-Bump with Bump comparison, the Level 1 effects of first-time experiences and emotional valence were both significant and negative, showing that Post-Bump memories were less likely to describe first-time experiences (OR: 0.84) and included emotionally less positive memories compared to Bump memories (OR: 0.92). The effect of importance became statistically significant as well but positive, implying that PostBump memories include more memories rated as important compared to Bump memories (OR: 1.23). On Level 2, the effects of first-time experiences and importance became significant again. Participants who showed higher proportions of autobiographical memories describing first-time experiences reported fewer memories from the Post-Bump category than from the Bump category (ORs: 0.03). In contrast, participants who reported more important memoires recalled more memories from the Post-Bump compared to the Bump category (OR: 5.07).

\section{Commonality analysis}

In order to decompose the variance accounted for in the outcome variables Pre-Bump (vs. Bump) and Post-Bump (vs. Bump) into portions uniquely attributable to first-time experiences, emotional valence, and importance, a commonality analysis was conducted. Note that "variance accounted for" is an expression used here for convenience only. Because the two outcome variables are dichotomous (Pre-Bump vs. Bump and Post-Bump vs. Bump), it is difficult to define the residual variance. What is possible, however, is to define the residual variance on the latent (or link) scale, that is, on the logit scale in our case. One has to keep in mind that such a definition of the residual variance is specific to the error distribution and the link function used in the analysis (see Nakagawa \& Schielzeth, 2013).

Results are shown in Fig. 2, separately for the withinperson and between-person effects and the two parts of the multinomial mixed-effects model. Unique portions are depicted as black bars, common portions (variance accounted for shared with the other predictor variables) as gray bars, the total amount of variance explained as striped bars.

Several things are noteworthy. First, while the total amount of variance accounted for in the within-person Pre-Bump model was approximately $13 \%$ for all three predictor variables together (see striped bar in the left half of the upper panel of Fig. 2), unique effects compared to common effects were relatively small (around one-quarter to one-fifth of the total effect). This implies that within-person the effects of first-time experiences, emotional valence, and importance in explaining which autobiographical memories fall into the Pre-Bump (as opposed to the Bump) largely overlap. Importantly, if one would only consider one of these predictor variables separately, one would have the incorrect impression that each predictor explains approximately $10 \%$ of variance (which represents the unique effect and the common effect). 


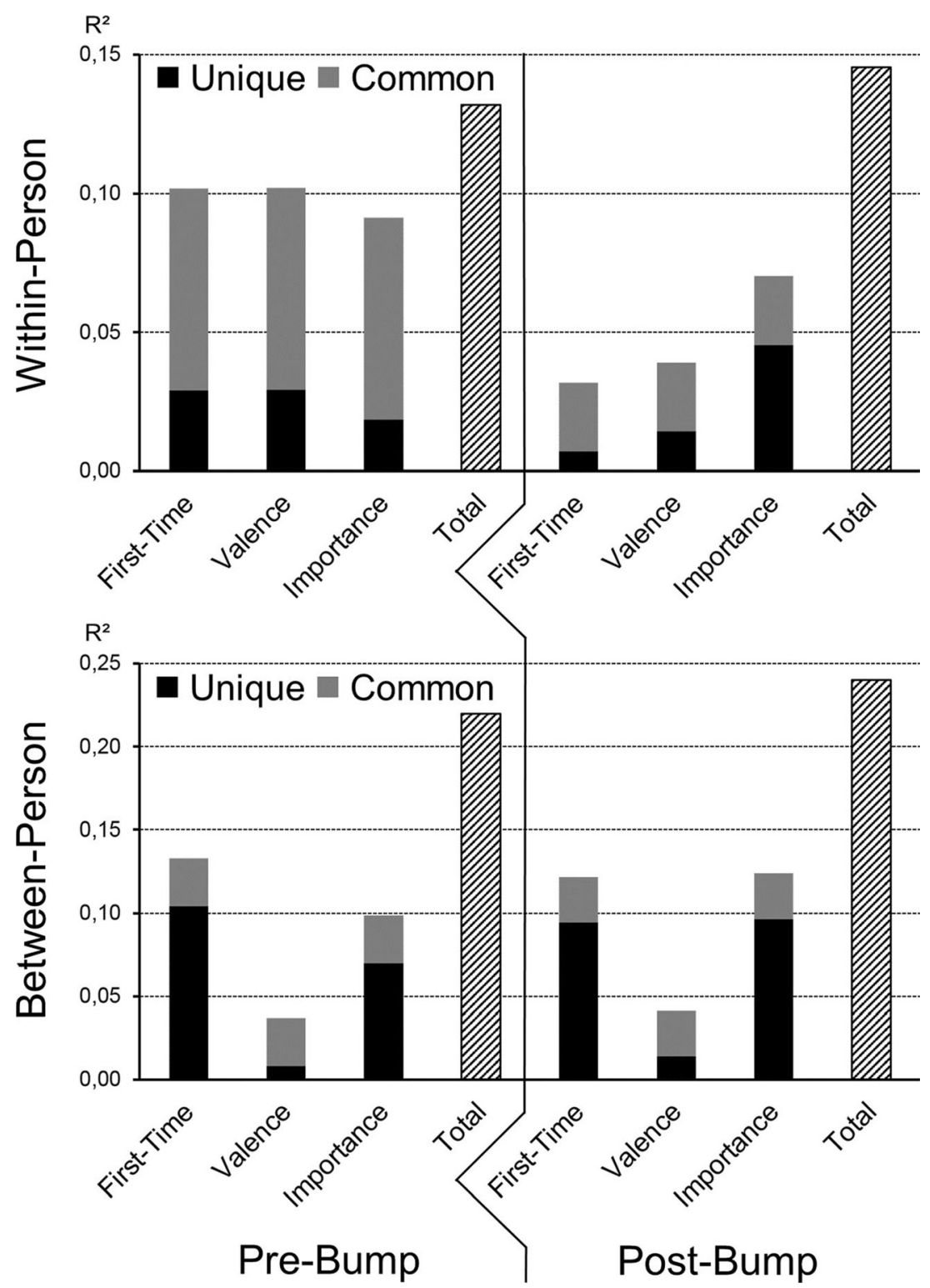

Fig. 2 Results of the commonality analyses. Predictor variables are shown on the abscissa, the amount of $\mathrm{r}^{2}$ (on the logit scale) on the ordinate. Black bars indicate unique contributions of the predictor variables, gray bars indicate contributions common with the other

predictor variables. Upper panel: within-person model. Lower panel: between-person model. Left half: pre-bump versus bump model. Right half: post-bump versus bump model

Second, in the within-person Post-Bump model, the total amount of explained variance was $15 \%$. However, the amount of explained variance differed markedly between predictor variables (see right half of the upper panel in Fig. 2). While first-time experiences uniquely accounted for about $0.7 \%$ of variance, for valence this effect was approximately twice as large (1.4\%), while for importance the unique effect was $4.5 \%$. The common effect accounted for $2.5 \%$ of variance. Thus, only for importance the common effect was smaller than the unique effect. As with the Pre-Bump model, predictors showed considerable overlap and only importance appears to make a substantial unique contribution in explaining why an autobiographical memory falls into the Post-Bump period of life and not into the Bump.

Third, for the between-person models, a different picture emerged. Apart from valence, for first-time experiences and importance the unique parts dominated the amount of variance accounted for. Both in the Pre- and Post-Bump models, firsttime experiences uniquely accounted for approximately $10 \%$ of variance. Similarly, importance uniquely explained $7 \%$ of variance in the Pre-Bump model and $10 \%$ in the Post-Bump model. Common effects in both models accounted for approximately $3 \%$ of variance. Thus, apart from emotional valence, predictor variables (first-time experiences and importance) are 
much more distinct in their effects in the between-person model than in the within-person model. The total amount of explained variance between persons (23\% and 24\%) was considerably higher than in their within-person counterparts.

From these findings, we conclude that within-person predictor variables that represent different theoretical accounts (1) show relatively large overlap and (2) together, slightly better differentiate between autobiographical memories falling into the PostBump period versus the Bump period (as indicated by a higher $\mathrm{R}^{2}$ ). Moreover, on the between-person level (3) valence does hardly explain variance, whereas (4) first-time experiences and importance uniquely account for substantial portions of variance.

\section{Discussion}

Explanatory accounts of the reminiscence bump have often focused on what distinguishes autobiographical memories from the reminiscence bump period from adolescence and young adulthood from those from other life periods. In examining the explanatory power of different explanatory accounts, one has to take into consideration that (1) different accounts may lead to similar predictions and that (2) different predictors are not mutually exclusive. In fact, the mechanisms postulated in different explanatory accounts may simultaneously contribute to the formation of the reminiscence bump (e.g., Demiray et al., 2009; Munawar et al., 2018). In the present study, we used a multilevel multinomial model introduced by Zimprich and Wolf (2018) in conjunction with commonality analysis to examine the predictive power of four different accounts of the reminiscence bump simultaneously, namely the cognitive explanatory account, the identity-formation account, the life-script account and the life-story account.

\section{Predictors of the reminiscence bump}

Based on the aforementioned accounts, we expected the reminiscence bump to be associated with greater numbers of firsttime experiences, as well as with memories that were perceived as relatively more important for a person's life and emotionally more positive than memories from other life periods. As expected, we found all three predictor variables to meaningfully distinguish between memories from the reminiscence bump period and memories from the earlier, respectively later, life periods. However, not all effects went into the hypothesized direction.

For instance, based on the cognitive explanatory account (e.g., Pillemer, 2001; Rubin et al., 1998), we expected that an autobiographical memory describing a first-time experience would be more likely to stem from the reminiscence bump period than from other life periods (Level 1 prediction). At the same time, we expected that participants who reported greater proportions of autobiographical memories describing first-time experiences would show a more pronounced reminiscence bump - as would be indicated by (relatively) more autobiographical memories falling into the reminiscence bump period (Level 2 prediction). The effects of first-time experiences were statistically significant in both the pre-bump and postbump model as well as on the within- (Level 1) and betweenperson level (Level 2). This indicates that the number of memories referring to first-time experiences differs between the reminiscence bump and the life periods before and after. Based on these findings, one may conclude that novelty is indeed a critical factor in forming the reminiscence bump. However, whereas first-time experiences were more likely to stem from the reminiscence bump period than from a later life period (postbump), they were even more likely to stem from the life period before the reminiscence bump (pre-bump). This is in line with findings reported by Jansari and Parkin (1996). Also using the cue word technique, the authors found that middle-aged adults reported greater proportions of first-time experiences from the first two decades of life (age 0-20 years) compared to memories from the last 10-20 years of their life. Taken together, these findings suggest that the likelihood of recalling first-time experiences simply decreases across the lifespan - which seems logical, given that the chance of experiencing first-time events decrease with age. Consequently, an explanatory account only focusing on the novelty of an event can explain why memories from the reminiscence bump differ from memories from succeeding life periods. However, it is not suited to explain the increase in the number of memories from the second decade of life, that is, the lower bound of the reminiscence bump.

The reversed pattern occurred regarding the perceived importance of memories for participants' lives: The prediction that the reminiscence bump would be associated with higher ratings of importance was only true for the comparison between memories from the bump and memories from an earlier life period (i.e., the pre-bump category). Memories from the life periods after the reminiscence bump, however, were rated as even more important. We found the same pattern on the level of individuals: Participants, who, on average, perceived their memories as more important, reported more memories from the reminiscence bump than from the earlier life period, but fewer memories from the reminiscence bump compared to the succeeding life periods. This finding challenges explanatory accounts that solely draw on the importance of memories for an individuals' life in order to explain the reminiscence bump phenomenon. For instance, the identity-formation account argues that memories from the life period in which a person's identity was developed remain highly accessible (and are recalled frequently), because they are still considered as important for this person's life (Conway, 2005; Rathbone et al., 2008). Based on our present findings, this account appears to be useful in explaining why individuals recall more memories from their adolescent and young adult years in comparison to memories from their early childhood - which could indeed be explained by identity formation. However, the present results suggest that events experienced after the 
reminiscence bump are perceived as even more important for an individual's life. Although the identity-formation account does not predict that self-defining experiences can only occur during the reminiscence bump period, an account solely focusing on the importance of memories for an individual's life cannot entirely explain the reminiscence bump phenomenon.

The life-script account (Rubin \& Berntsen, 2003), in contrast, builds upon the (cultural) importance of autobiographical memories but additionally predicts an association between the emotional valence of autobiographical memories and the reminiscence bump: Life scripts represent idealized life stories and, thus, include more events that are considered positive (i.e., marriage). As a result, life scripts are more likely to activate memories of positive experiences (e.g., Zaragoza Scherman, 2013). In line with this prediction, we found the reminiscence bump to be associated with emotionally more positive memories compared to memories from the life periods before and after the reminiscence bump. Additionally, participants who reported, on average, emotionally more positive memories recalled more autobiographical memories from the reminiscence bump period than participants who reported emotionally less positive memories (Level 2). Thus, the predicted association between the emotional valence of autobiographical memories and the reminiscence bump was substantiated at the level of the remembered events as well as at the level of the person remembering these events, and equally hold for the comparison with an earlier and later life periods. Thus, of the three explanatory variables used in the present study, only emotional valence was clearly associated with the reminiscence bump phenomenon implying that the bump is characterized by emotionally more positive memories. Again, this does not imply that positive experiences can only occur during the reminiscence bump period, but that memories from this life period are rated as relatively more positive than memories from other life periods.

Taken together, the present findings indicate that all three explanatory variables can meaningfully distinguish between memories from the reminiscence bump and memories from the life periods before and after the bump. Although the present study is not the first to argue that different mechanisms may contribute simultaneously to the reminiscence bump phenomenon (e.g., Demiray et al., 2009; Ece \& Gülgöz, 2017), this has not been tested adequately yet, that is, by using a multivariate approach that takes into account that memories are nested within individuals. Thus, the present results contribute to the literature by showing (1) that all three explanatory variables are associated with the reminiscence bump even after controlling for possible effects of the other two, and (2) that these findings equally hold on the level of memories as well as on the level of individuals. Regarding different explanatory accounts, our results indicate that accounts focusing on the emotional valence of autobiographical memories (i.e., the life-script account and the life-story account) are well suited to distinguish autobiographical memories from the reminiscence bump period from memories of an earlier and later life period. The explanatory variables derived from the cognitive account and the identity-formation account appear also useful in describing bump memories, but only in separating them from either an earlier or later life period, not from both. This does not necessarily mean that these accounts cannot explain the reminiscence bump phenomenon. What needs to be examined further is whether these different characteristics need to be combined in order to explain the reminiscence bump phenomenon. To give an example, the reminiscence bump may not simply be characterized by first-time experiences or by memories rated high in importance, but rather occurs for memories referring to first-time experiences and, at the same time, being perceived as important for an individual's life. One way to identify the extent to which different explanatory variables overlap in explaining the reminiscence bump phenomenon is the use of commonality analysis.

\section{Commonality analysis}

Results of the commonality analysis are informative with respect to both the absolute size and the relative size of effects. In what follows, we will discuss these results for within and between-person effects separately.

Within-person effects Regarding the comparison of memories from the reminiscence bump and memories from an earlier life period, we found that first-time experiences, importance and emotional valence together accounted for approximately $13 \%$ of variance (on the logit scale). According to the standards defined by Cohen (1988), this would correspond to a mediumsized effect. Thus, the explanatory variables used in the present study meaningfully distinguish between memories from the reminiscence bump and memories from an earlier life period. Regarding the relative size of unique and shared effects, the three explanatory variables showed large overlap in their effects, implying that common effects were considerably larger than unique effects. This finding has important theoretical but also methodological implications. First, it clearly demonstrates the benefits of a multivariate analysis, because the amount of total variance accounted for $(13 \%)$ is considerably smaller than the sum of the variances accounted for by individual predictor variables considered separately (approx. 29\%). Thus, examining different explanatory variables separately may overestimate the effect of each predictor variable. A meaningful examination of different explanatory accounts of the reminiscence bump needs to be based on a multivariate analytical approach. Second, these findings demonstrate that explanatory variables overlap, which not simply implies that the mechanisms postulated in different explanatory accounts may simultaneously contribute to the formation of the reminiscence bump (e.g., Demiray et al., 2009; Munawar et al., 2008) but that they are somewhat interchangeable. In other words, event-characteristics such as novelty, 
importance, and emotional valence appear to be equally suited to distinguish between memories from the reminiscence bump and memories from an earlier life period. In the present study, we found that memories from the reminiscence bump were characterized by higher ratings of importance and positive valence, but these important and positive memories were less likely to refer to first-time experiences - as compared to memories from an earlier life period.

A similar picture emerged for the bump versus post-bump comparison: Predictor variables accounted for approximately $15 \%$ of variance (on the logit scale), which again corresponds to a medium-sized effect (Cohen, 1988). Regarding the relative size of unique and shared effects, the three explanatory variables also overlapped in their effects. With respect to novelty and emotional valence, common effects were considerably larger than unique effects. This implies that the reminiscence bump is associated with greater numbers of first-time experiences, which are, at the same time, perceived as emotionally more positive than memories from the succeeding life period. A different picture emerged for the perceived importance of memories, because here, the size of the unique effect of importance was larger than the shared effect. Thus, the degree to which an autobiographical memory is perceived as important appears to separate best between bump and post-bump memories (at least on a relative scale, that is, compared to the other two predictor variables). One has to keep in mind, though, that the effect of importance went in the opposite direction as one would assume based on the identity-formation, for instance (see also the lifescript account and the life-story account). Taken together, memories from the reminiscence bump were characterized by a greater number of first-time events that were also more likely to be perceived as emotionally more positive than memories from the succeeding life period. In addition, memories from the reminiscence bump were overall perceived as less important.

To sum up, regarding within-person effects, we found the explanatory variables used in the present study to clearly overlap. This makes it difficult to empirically separate between the different explanatory accounts we considered. Transferred back to a theoretical level of explaining the reminiscence bump, this implies that the explanatory variables derived from different accounts are not distinctive enough to lead to empirically separable findings. This could be seen as support for the life-story account (Glück \& Bluck, 2007), which acknowledges the influence of several explanatory variables (Demiray et al., 2009). In addition, the present findings suggest that explanatory variables are not equally suited to distinguish memories from the reminiscence bump from both an earlier and a later life period: Whereas first-time experiences were most frequent in early childhood, memories from after the reminiscence bump period were considered as being most important for an individual's life. Thus, a comprehensive account of the reminiscence bump may not only consider different explanatory variables simultaneously, but may also provide different predictions regarding the onset and the offset of the reminiscence bump.
Between-person effects At the between-person level, predictor variables accounted for approximately $23 \%$ of the variance (on the logit scale) in the pre-bump versus bump comparison, which comes close to a large effect (Cohen, 1988). Apart from emotional valence, unique effects were larger than shared effects, implying that on the between-person level predictor variables were more distinct than on the within-person level. Even after controlling for (random) individual intercept differences (that is, individual differences in the total number of memories falling into the pre-bump, bump, and post-bump categories), there were individual differences in how many first-time experiences or important memories participants reported. These individual differences are, in turn, strong predictors of how many memories (relatively seen) a participant reports from the pre-bump, bump, and post-bump categories. Again, however, first-time experiences showed an effect opposite to what would have been expected based on the cognitive account (cf. Rubin et al., 1998). The bump versus post-bump comparison at the between-person level, where predictor variables accounted for approximately $24 \%$ of variance (on the logit scale), led to similar findings. With the exception of emotional valence, unique effects were larger than shared effects. However, importance showed the opposite effect compared to what would have been expected based on the identity-formation, life-script and life-story accounts.

These findings have, in our view, important theoretical implications. First, although the explanatory accounts considered in this study have been developed to account for withinperson effects, our results show that their effects are larger on the between-person level. Albeit it is straightforward and meaningful to derive hypotheses from the explanatory accounts for the between-person level (see above), only a few studies have done so. Wolf and Zimprich (2016a), for instance, examined individual differences in the distribution of autobiographical memories. They found that individuals who reported higher proportions of first-time experiences showed an earlier and more pronounced reminiscence bump (as indicated by a smaller mean age and a smaller scale). Of course, genuine between-person hypotheses could also be developed. To give an example, if the reminiscence bump occurs because autobiographical memories from an individual's youth are perceived as important for his or life-story and identity - as proposed in the identity-formation or the life-story account, the reminiscence bump should be more pronounced for individuals with a clear and temporarily stable self-concept. In the aforementioned study by Wolf and Zimprich (2016a), the authors found higher ratings of self-concept clarity to be associated with a more pronounced reminiscence bump that was located earlier compared to the distribution of individuals with a less clear and temporarily less stable self-concept (see Zimprich \& Wolf, 2016, for similar findings regarding the perceived sense of coherence). Apart from these studies, the examination of between-person differences in the distribution of autobiographical memories represents a neglected area of 
research, which seems unwarranted given our results. In fact, given the fact that the explanatory variables used in the present study showed larger total and unique effects (except for emotional valence) on the between-person level, a comprehensive account of the reminiscence bump may also consider individual differences in autobiographical memory.

\section{Limitations and future directions}

One has to keep in mind that the results reported here may not completely generalize to the generation of autobiographical memories under different conditions. While in the present study cue words were used, results might look different if participants are asked to report important memories only, for example (see Glück \& Bluck, 2007). Note that different cueing technique may lead to different result regarding the location and the shape of the reminiscence bump. Whereas wordcued memories tend to show an early peak in their distribution located roughly between the ages of 9 and 23 years (cf. Koppel \& Berntsen, 2015), more restrictive cueing techniques that ask participants to recall memories of a certain kind (e.g., important or positive memories) may result in a later and more pronounced reminiscence bump located in the third decade of life. Nevertheless, both cueing techniques lead to an increase in memories from participants' youth, respectively young adult years. The occurrence of a reminiscence bump can, therefore, not only be explained by different retrieval strategies but may additionally be due to influential factors related to the encoding and retention of autobiographical memories (see Ece \& Gülgöz, 2017 for a similar argument). Still, a multivariate analysis that takes two levels of analysis and several explanatory variables simultaneously into account may provide new insights. Moreover, our analysis approach easily allows for the inclusion of different ways to elicit autobiographical memories, which could enter the analyses as a design variable. Doing so would then make a direct and methodologically sound comparison of different ways of asking participants for autobiographical memories possible - something that is still missing in the literature.

Of course, alternative approaches have been suggested in order to analyze the reminiscence bump phenomenon. For instance, Zimprich and Wolf (2016a; Wolf \& Zimprich, 2016b) used mixed logitnormal regression to examine individual differences in the distribution of autobiographical memories. Although this approach is well suited to examine individual differences in the location and the shape of the reminiscence bump, one cannot define the age boundaries of the reminiscence bump and, consequently, cannot compare bump memories with memories from other life periods. The multilevel multinomial model that we have used in the present study, in contrast, has the advantage that it does not rely on specific distributional assumptions (Zimprich \& Wolf, 2018).
The drawback, however, is that category boundaries are fixed (e.g., for all participants we assume that the age boundary between pre-bump and bump is at the age of 10 years). However, in the present study, these fixed age boundaries are in line with most of previous studies that reported a reminiscence bump in the distribution of word-cued memories (see Koppel \& Berntsen, 2015).

In the data from out study, no recency effect was present because participants were asked to report autobiographical memories older than 10 years (see Footnote 2). One may wonder how results would look like if recent autobiographical memories were included. Naturally, these more recent autobiographical memories would all fall into the post-bump period in an older sample (like the one in our study), which would only change the comparison between bump and post-bump autobiographical memories. However, a more adequate way to include recent memoires might consist of adding a fourth category, e.g., Category D (Recency), in the multinomial mod$\mathrm{el}$ - which was beyond the scope of the present study.

\section{Conclusion}

The present study contributes to the literature on the reminiscence bump by providing new answers to the frequently asked question of what characterizes the reminiscence bump in autobiographical memories. Although it is widely understood that different explanatory accounts are not mutually exclusive, the present study is the first that systematically and directly compared the predictive power of different accounts simultaneously using multilevel multinomial models in conjunction with commonality analysis. Our findings provide empirical support for the assumption that different mechanisms indeed contribute simultaneously to the reminiscence bump phenomenon - which renders it important to (1) examine more than one explanatory variable in studies on autobiographical memory and (2) to investigate their joint effects.

Our results of large shared effect also correspond to the lifestory account (cf. Demiray et al., 2009), which takes a more integrative view on the reminiscence bump. Additionally, the present results indicate that individual differences play an important role in autobiographical remembering, which could be integrated into an explanatory account that focusses on what distinguishes us from another, that is, our individual life story. However, a more thorough examination of the life-story account would have required the inclusion of additional predictor variables (e.g., subjective control). Of course, future research is needed to test the reliability of our results. Moreover, the predictor variables considered in the present study represent but a selection of possible explanatory variables and our results suggest that there is room for more explanatory variables. Complementary predictors could be derived from the four explanatory accounts included in the present study but also from 
explanatory accounts not considered here (e.g., the cognitive abilities account; Janssen, Kristo, Rouw, \& Murre, 2015).

Open Practices Statements An excerpt of the datasets generated during and/or analyzed during the current study as well as the SAS code for fitting the multilevel multinomial models are included in this published article (and its Supplementary Material files).

\section{References}

Alea, N., Ali, S., \& Marcano, B. (2014). The bumps in Trinidadian life: Reminiscence bumps for positive and negative life events. Applied Cognitive Psychology, 28, 174-184.

Akaike, H. (1974). A new look at the statistical model identification. IEEE Transactions on Automatic Control, 19, 716-723.

Arnett, J. J. (2000). Emerging adulthood: A theory of development from the late teens through the twenties. American Psychologist, 55, 469480.

Berntsen, D., \& Rubin, D. C. (2002). Emotionally charged autobiographical memories across the lifespan: The recall of happy, sad, traumatic, and involuntary memories. Psychology and Aging, 17, 636-652.

Berntsen, D., \& Rubin, D. C. (2004). Cultural life scripts structure recall from autobiographical memory. Memory \& Cognition, 32, 427-442.

Bjork, R. A. (1975). Retrieval as a memory modifier: An interpretation of negative recency and related phenomena. In Robert L. Solso (Ed.), Information Processing and Cognition: The Loyola Symposium (pp. 123-144). Hillsdale, NJ: Lawrence Erlbaum.

Bryk, A. S., \& Raudenbush, S. W. (1992). Hierarchical linear models. Newbury Park, CA: Sage.

Cohen, J. (1988). Statistical power analysis for the behavioral sciences (2nd ed.). Hillsdale, NJ: Lawrence Erlbaum

Conway, M. A. (2005). Memory and the self. Journal of Memory and Language, 53, 594-628.

Conway, M. A., \& Haque, S. (1999). Overshadowing the reminiscence bump: Memories of a struggle for independence. Journal of Adult Development, 6, 35-44.

Conway, M. A., Wang, Q., Hanyu, K., \& Haque, S. (2005). A crosscultural investigation of autobiographical memory: On the universality and cultural variation of the reminiscence bump. Journal of Cross-Cultural Psychology, 36, 739-749.

Craik, F. I. M., \& Lockhart, R. S. (1972). Levels of processing: A framework for memory research. Journal of Verbal Learning \& Verbal Behavior, 11, 671-684.

Demiray, B., Gülgöz, S., \& Bluck, S. (2009). Examining the life-story account of the reminiscence bump: Why we remember more from young adulthood. Memory, 17, 708-723.

Diggle, P. J., Heagerty, P., Liang, K.-Y., \& Zeger, S. L. (2002). Analysis of longitudinal data. Oxford. Oxford University Press.

Ebner, N. C., Freund, A. M., \& Baltes, P. B. (2006). Developmental changes in personal goal orientation from young to late adulthood: From striving for gains to maintenance and prevention of losses. Psychology and Aging, 21, 664-678.

Ece, B., \& Gülgöz, S. (2014). The impact of suppressing the typical life events on the reminiscence bump. Applied Cognitive Psychology, $28,702-710$.

Ece, B., \& Gülgöz, S. (2017). Is the road still bumpy without the most frequent life events? Applied Cognitive Psychology, 31, 326-339.

Erikson, E. H. (1950). Child and society. New York, NY: Norton.
Eysenck, M. W., \& Eysenck, M. C. (1980). Effects of processing depth, distinctiveness, and word frequency on retention. British Journal of Psychology, 71, 263-274.

Fitzgerald, J. M. (1988). Vivid memories and the reminiscence phenomenon: The role of a self narrative. Human Development, 31, 261273.

Fitzgerald, J. M. (1996). The distribution of self-narrative memories in younger and older adults: Elaborating the self-narrative hypothesis. Aging, Neuropsychology and Cognition, 3, 229-236.

Glück, J., \& Bluck, S. (2007). Looking back across the life span: A lifestory account of the reminiscence bump. Memory \& Cognition, 35, 1928-1939.

Havighurst, R. J. (1948). Developmental tasks and education. New York, NY: McKay.

Hedeker, D. (2003). A mixed-effects multinomial logistic regression model. Statistics in Med-icine, 21, 1433-1446.

Hedeker, D., du Toit, S. H. C., Demirtas, H., \& Gibbons, R. D. (2018). A note on marginalization of regression parameters from mixed models of binary outcomes. Biometrics, 74, 354-361.

Hedeker, D., \& Gibbons, R. D. (2006). Longitudinal data analysis. New York: Wiley.

Holbrook, M. B., \& Schindler, R. M. (1989). Some exploratory findings on the development of musical tastes. Journal of Consumer Research, 16, 119-124.

Hyland, D. T., \& Ackerman, A. M. (1988). Reminiscence and autobiographical memory in the study of the personal past. Journal of Gerontology, 43, 35-39.

Jansari, A., \& Parkin, A. J. (1996). Things that go bump in your life: Explaining the reminiscence bump in autobiographical memory. Psychology and Aging, 11, 85-91.

Janssen, S. M. J., Chessa, A. G., \& Murre, J. M. J. (2005). The reminiscence bump in autobiographical memory: Effects of age, gender, education, and culture. Memory, 13, 658-668.

Janssen, S. M. J., Chessa, A. G., \& Murre, J. M. J. (2007). Temporal distribution of favourite books, movies, and records: Differential encoding and re-sampling. Memory, 15 (7), 755-767.

Janssen, S. M. J., Kristo, G., Rouw, R., \& Murre, J. M. J. (2015). The relation between verbal and visuospatial memory and autobiographical memory. Consciousness and Cognition, 31, 12-23.

Janssen, S. M. J., \& Murre, J. M. J. (2008). Reminiscence bump in autobiographical memory: Unexplained by novelty, emotionality, valence, or importance of personal events. The Quarterly Journal of Experimental Psychology, 61, 1847-1860.

Janssen, S.M. J., Rubin, D. C., \& St. Jacques, J. L. (2011). The temporal distribution of autobiographical memory: Changes in reliving and vividness over the life span do not explain the reminiscence bump. Memory \& Cognition, 39, 1-11.

Kawasaki, Y., Janssen, S. M. J., \& Inoue, T. (2011). Temporal distribution of autobiographical memory: Uncovering the reminiscence bump in Japanese young and middle-aged adults. Japanese Psychological Research, 53, 86-96.

Koppel, J., \& Berntsen, D. (2015). The peaks of life: The differential temporal locations of the reminiscence bump across disparate cueing methods. Journal of Applied Research in Memory and Cognition, 4, 66-80.

Larsen, S. F. (1996). Memorable books: Recall of reading and its personal context. In R. J. Kreuz and M. S. MacNealy (Eds.), Empirical approaches to literature and aesthetics (pp. 583-600). Norwood, NJ: Ablex.

Munawar, K., Kuhn, S. K., \& Haque, S. (2018). Understanding the reminiscence bump: A systematic review. PLOS ONE 13(12): e0208595.

Nakagawa, S. \& Schielzeth, H. (2013). A general and simple method for obtaining $\mathrm{R}^{2}$ from generalized linear mixed effects models. Methods in Ecology and Evolution, 4, 133-142. 
Nimon, K., \& Reio, T. G. (2011). Regression Commonality Analysis: A technique for quantitative theory building. Human Resource Development Review, 10, 329-340.

Pillemer, D. B. (2001). Momentous events and the life story. Review of General Psychology, 5, 123-134.

Prebble, S. C., Addis, D. R., \& Tippett, L. J. (2013). Autobiographical memory and sense of self. Psychological Bulletin, 139, 815-840.

Ray-Mukherjee, J., Nimon, K., Mukherjee, S., Morris, D. W., Slotow, R., \& Hamer, M. (2014). Using commonality analysis in multiple regressions: A tool to decompose regression effects in the face of multicollinearity. Methods in Ecology and Evolution, 5, 320-328.

Rathbone, C. J., O'Connor, A. R., \& Moulin, C. J. A. (2017). The tracks of my years: Personal significance contributes to the reminiscence bump. Memory \& Cognition, 45, 137-150.

Rathbone, C. J., Moulin, C. J. A., \& Conway, M. A. (2008). Self-centered memories: The reminiscence bump and the self. Memory \& Cognition, 36, 1403-1414.

Robinson, J. A. (1992). First experience memories: Contexts and function in personal histories. In M. A. Conway, D. C. Rubin, H. Spinnler, \& W. A. Wager (Eds.), Theoretical perspectives on autobiographical memory (pp. 223-239). Dordrecht, The Netherlands: Kluwer Academic Publishers.

Rubin, D. C., \& Berntsen, D. (2003). Life scripts help to maintain autobiographical memories of highly positive, but not highly negative, events. Memory \& Cognition, 31, 1-14.

Rubin, D. C., Rahhal, T. A., \& Poon, L. W. (1998). Things learned in early adulthood are remembered best. Memory \& Cognition, 26, 319.

Rubin, D. C., \& Schulkind, M. D. (1997a). Distribution of important and word-cued autobiographical memories in 20-, 35-, and 70-year-old adults. Psychology and Aging, 12, 524-535.

Rubin, D. C., \& Schulkind, M. D. (1997b). The distribution of autobiographical memories across the lifespan. Memory \& Cognition, 25, 859-866.

Rubin, D. C., Wetzler, S. E., \& Nebes, R. D. (1986). Autobiographical memory across the adult lifespan. In D. C. Rubin (Ed.), Autobiographical memory (pp. 202-221). Cambridge: Cambridge University Press.

SAS Institute Inc. (2014). SAS/STAT® 13.2 User's Guide. Cary, NC: SAS Institute Inc.

Schwarz, G. E. (1978). Estimating the dimension of a model. Annals of Statistics, 6, 461-464.

Stroup, W. W. (2013). Generalized linear mixed models: Modern concepts, methods and applications. Boca Raton, FL: CRC Press.
Võ, M. L.-H., Conrad, M., Kuchinke, L., Hartfeld, K., Hofmann, M. F., \& Jacobs, A. M. (2009). The Berlin Affective Word List Reloaded (BAWL-R). Behavior Research Methods, 41, 534-538.

Vrieze, S. I. (2012). Model selection and psychological theory: A discussion of the differences between the Akaike Information Criterion (AIC) and the Bayesian Information Criterion (BIC). Psychological Methods, 17, 228-243.

Webster, J. D., \& Gould, O. (2007). Reminiscence and vivid personal memories across adulthood. The International Journal of Aging and Human Development, 64, 149-170.

Wolf, T., \& Zimprich, D. (2016a). How can individual differences in autobiographical memory distributions of older adults be explained? Memory, 24, 1287-1299.

Wolf, T., \& Zimprich, D. (2016b). The distribution and the functions of autobiographical memories: Why do older adults remember autobiographical memories from their youth? European Journal of Ageing, 13, 241-250.

Zaragoza Scherman, A. (2013). Cultural life script theory and the reminiscence bump: A reanalysis of seven studies across cultures. Nordic Psychology, 65, 103-119.

Zaragoza Scherman, A., Salgado, S., Shao, Z., \& Berntsen, D. (2015). Life span distribution and content of positive and negative autobiographical memories across cultures. Psychology of Consciousness: Theory, Research, and Practice, 2, 475-489.

Zimprich, D. (2018). Individual differences in the reminiscence bump of very long-term memory for popular songs in old age: A non-linear mixed model approach. Psychology of Music. Advance online publication. https://doi.org/10.1177/0305735618812199

Zimprich, D., \& Kurtz, T. (2013). Individual differences and predictors of forgetting in old age: The role of processing speed and working memory. Aging, Neuropsychology, \& Cognition, 20, 195-219.

Zimprich, D., \& Wolf, T. (2016a). Modeling individual differences in autobiographical memory distributions using mixed logitnormal regression. Applied Cognitive Psychology, 30, 360-374.

Zimprich, D., \& Wolf, T. (2016b). The distribution of memories for popular songs in old age: An individual differences approach. Psychology of Music, 44, 640-657.

Zimprich, D., \& Wolf, T. (2018). Leveling up the analysis of the reminiscence bump in autobiographical memory: A new approach based on multilevel multinomial models. Memory \& Cognition, 46, 11781193.

Publisher's note Springer Nature remains neutral with regard to jurisdictional claims in published maps and institutional affiliations. 